capillaries. About subpapillary venous plexus in SCL: 7 (32\%) were prominent and only one had capillary hemorrhage. In contrast, none of the controls presented these alterations. ( $p 0.0$ ).

According to the 3 NVC patterns the following averages were observed in the LVC:

Early Pattern (n: 8): diameter 36.93 $\pm 10.84 \mu \mathrm{m}$; Long $191.21 \pm 47.58 \mu \mathrm{m}$; Density $18.12 \pm 5.33 \mu \mathrm{m}$.

Active Pattern (n: 11): diameter 48.80 $\pm 14.80 \mu \mathrm{m}$; Length 184.11 $\pm 29.91 \mu \mathrm{m}$; Density $16.72 \pm 3.25 \mu \mathrm{m}$.

Late Pattern ( $\mathrm{n}: 3$ ): diameter $36.27 \pm 10$; Long $178.03 \pm 28.8 \mu \mathrm{m}$, density $19.33 \pm 4.16$ $\mu \mathrm{m}$.

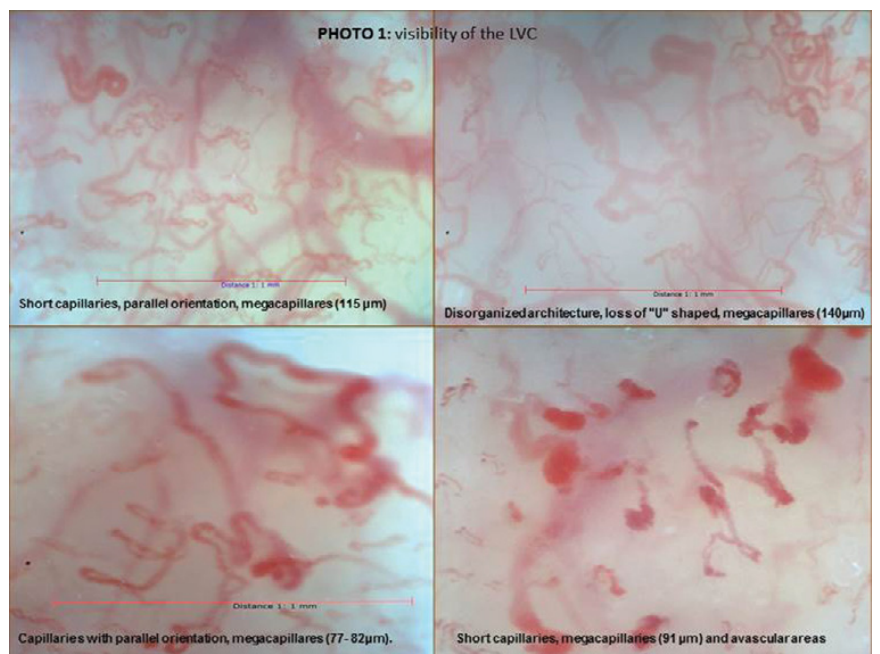

Conclusions: We found that LVC in SCL patients show significant microvascular changes with respect to $\mathrm{HC}$. In addition, all NVC patterns described in SCL showed similar alterations in LVC. Therefore, LVC could be a complementary or alternative method to NVC since it is easily accessible, has good visibility and is not influenced by local mechanical or chemical stimuli that can affect the nail bed. References:

[1] W Grassi et al. Ann Rheum Dis 1993; 52 (8): 564-569.

[2] M. Cutolo et all. Rheumatology 2006; 45: 43-46.

Disclosure of Interest: None declared

DOI: 10.1136/annrheumdis-2017-eular.4329

\section{FRI0636 ENDOTHELIAL DYSFUNCTION IN SYSTEMIC LUPUS ERYTHEMATOSUS - A CASE-CONTROL STUDY AND AN UPDATED META-ANALYSIS AND META-REGRESSION}

A. Mak ${ }^{1}$, N.Y. Kow ${ }^{1}$, H. Schwarz ${ }^{2}$, L.L. Gong ${ }^{3}$, S.H. Tay ${ }^{1}$, L.H. Ling ${ }^{3}$ ${ }^{1}$ Medicine; ${ }^{2}$ Physiology; ${ }^{3}$ Cardiology, National University of Singapore, Singapore, Singapore

Background: Endothelium-dependent flow-mediated dilation (ED-FMD), a biophysical marker of endothelial dysfunction, is apparently impaired in patients with systemic lupus erythematosus (SLE). However, such observation is inconsistent because of the lack of standardization of the methodology of ED-FMD measurement and inclusion of patients with different comorbidities amongst different studies.

Objectives: Firstly, we evaluated if ED-FMD is indeed impaired in SLE patients naïve of cardiovascular disease and its traditional risk factors. Secondly, we aimed to determine if the putative contribution of SLE to endothelial dysfunction is in fact confounded by demographic-, disease- and treatment-related factors.

Methods: We assessed and compared the brachial artery ED-FMD (baED-FMD) using the Prosound Alpha-10 ultrasound system ${ }^{\circledR}$ between SLE patients without cardiovascular disease and cardiovascular risk factors and healthy controls $(\mathrm{HC})$ matched for age, gender and body mass index (BMI). Exclusions were pregnancy, a history hypertension, diabetes mellitus, chronic kidney disease, cardiovascular and cerebrovascular diseases, and statin therapy. SLE-related disease activity and organ damage in the SLE patients were assessed using SELENA-SLEDAI and SLICC/ACR DI, respectively. With inclusion of our own data from this case-control study, we performed a comprehensive meta-analysis of case-control studies which compared baED-FMD between SLE patients and HC by determining the effect size of baED-FMD as standard mean difference (SMD). Demographic and clinical factors associated with the effect size were explored by mixed-model meta-regression.

Results: Seventy one SLE patients and $71 \mathrm{HC}$ were studied, and there were 6 men in each group. The mean $\pm S D$ age and BMI of SLE patients and HC were $39.21 \pm 13.4$ and $40.37 \pm 12.9$ years, and $22.54 \pm 5.1$ and $22.86 \pm 4.2 \mathrm{~kg} / \mathrm{m}^{2}$, respectively. In SLE patients, the mean $\pm S D$ daily prednisolone dose, SELENASLEDAI and SLICC/ACR DI were $13.43 \pm 14.4 \mathrm{mg}, 6.52 \pm 5.4$ and $0.17 \pm 0.4$, respectively. SLE patients had significantly lower baED-FMD than $\mathrm{HC}(3.72 \pm 2.8 \%$ vs $4.63 \pm 3.1 \%, p=0.032)$. In the SLE group, no association between baED-FMD and age, gender, BMI, blood pressure, duration of SLE, serum C3, C4 and antidsDNA levels, SELENA-SLEDAI, SLICC/ACR DI and daily prednisolone dose was found. Similarly, no association was noted between baED-FMD and age, gender and $\mathrm{BMI}$ in the $\mathrm{HC}$ group. Meta-analysis of 25 case-control studies involving 1,313 SLE patients and $1,012 \mathrm{HC}$ with the random effects model revealed significantly lower baED-FMD in SLE patients compared to HC (SMD -1.077, $\mathrm{p}<0.001$ ) (see Fig for forest plot). The presence of diabetes mellitus $(p=0.04747)$, higher diastolic blood pressure $(p=0.04419)$ and renal involvement $(p=0.02721)$ were associated with more discrepant baED-FMD between both groups.

stcocrame

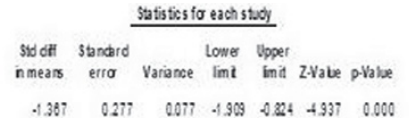

$\begin{array}{llllllll}\text { themad.2009 } & -1.367 & 0.277 & 0077 & 1.909 & 0.324 & +937 & 0.000\end{array}$

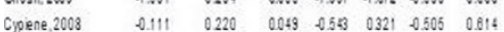
$\begin{array}{lllllllll}\text { Dhang. 2009 } & 0.858 & 0.198 & 0.035 & -1.027 & 0.289 & 3.493 & 0.000\end{array}$ $\begin{array}{llllllll}\text { Drang, 2009 } & 0.868 & 0.188 & 0.035 & -1.027 & 0.289 & 3.493 & 0.000 \\ \text { Vativelso, 2008 } & 0.885 & 0.307 & 0.094 & -1.487 & 0.283 & -2.880 & 0.004\end{array}$ $\begin{array}{lllllllll}\text { Sienungsson, } 2008 & 0.282 & 0.279 & 0.078 & 0.266 & 0.228 & 1.010 & 0.312\end{array}$ $\begin{array}{lllllllll}\text { Fiper.2007 } & 0.543 & 0.275 & 0.076 & -1008 & 0.003 & -1.971 & 0.049\end{array}$

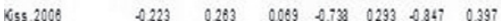

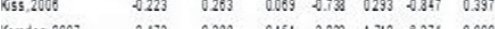

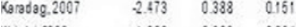
Whight $2006 \quad+1.000 \quad 0.300 \quad 0.094 \quad+80000.000 \quad 3.368 \quad 0.001$ Raggopalam,2004 $\quad 0.800 \quad 0.224 \quad 0.050 \quad+239 \quad 0.381 \quad 3.569 \quad 0.000$ $\begin{array}{llllllll}\text { Lima.2002 } \quad+307 & 0.228 & 0.051 & \$ .761 & -0.384 & 5.373 & 0.000\end{array}$ Hom

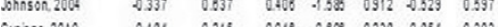

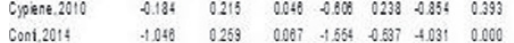
$\begin{array}{lllllllll}\text { Bassolou.2018 } & 0.302 & 0.114 & 0.013 & 0.099 & 0545 & 2.833 & 0.005\end{array}$

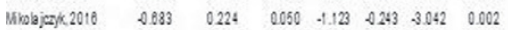
$\begin{array}{lllllllll}50 m e s .2012 & \$ .375 & 0.193 & 0.037 & \$ .753 & 0.004 & .9 .938 & 0.053\end{array}$

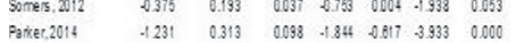
her: 2009

อ.8.

Snoer, 2015

Pramanik, 2011

Vake, 2013

Mak (presentstoy)

$\begin{array}{lllllll}0.000 & 0.281 & 0.068 & 0.511 & 0511 & 0.000 & 1.000 \\ 4939 & 0.484 & 0.215 & 5893 & 4031 & .0865 & 0.000\end{array}$

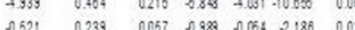

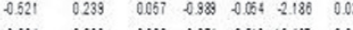

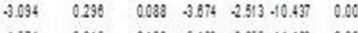

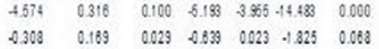

$\begin{array}{llllll}.077 \quad 0214 \quad 0048 & 1.497 & 0.057 & 8.025 & 0.000\end{array}$

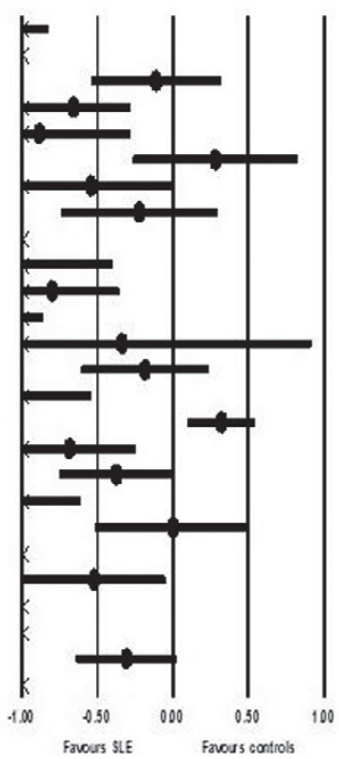

Conclusions: SLE patients naïve of cardiovascular disease and its traditional risk factors have impaired endothelial function. While SLE-related disease activity and organ damage are not apparently related to endothelial dysfunction, the presence of diabetes mellitus, renal disease and diastolic hypertension are potential contributors to endothelial dysfunction in SLE patients.

Disclosure of Interest: None declared

DOI: 10.1136/annrheumdis-2017-eular.4350

\section{FRI0637 DIAGNOSTIC PERFORMANCE OF THE NEW OMERACT CRITERIA FOR CPPD IDENTIFICATION BY US: CORRELATION WITH SYNOVIAL FLUID ANALYSIS}

A. Adinolfi ${ }^{1}$, V. Picerno ${ }^{1}$, A. Scanu ${ }^{2}$, C. Toscano ${ }^{1}$, C.A. Scirè ${ }^{3}$, G. Carrara ${ }^{4}$, B. Frediani ${ }^{1}$, A. lagnocco ${ }^{5}$, N. Damjanov ${ }^{6}$, L. Punzi ${ }^{2}$, G. Filippou ${ }^{1}$. ${ }^{1}$ University of Siena, Siena; ${ }^{2}$ University of Padova, Padova; ${ }^{3}$ University of Ferrara, Ferrara; ${ }^{4}$ SIR-Epidemiology Unit, Milan; ${ }^{5}$ University of Turin, Turin, Italy; ${ }^{6}$ University of Belgrade, Belgrade, Serbia

Background: Ultrasonography (US) has demonstrated to be an accurate tool for the diagnosis of calcium pyrophosphate deposition disease (CPPD) (1). Recently, the OMERACT "US in CPPD" subtask force, has created new definitions for CPPD identification by US, that demonstrated to be reliable at the knee joint, bridging a gap afflicting the old definitions (2). On the other hand, synovial fluid analysis (SFA) is considered to be an accurate and valid method for diagnosing CPPD.

Objectives: The aim of this study wasto evaluate the association between US, using for the first time the new OMERACT US criteria for CPPD, and SFA findings for identifying patients affected by CPPD.

Methods: We enrolled all the consecutive patients, aged more than 60 years old, referred to our outpatient clinic from September 2016 to December 2016, for knee pain and that presented knee effusion of any grade. Patients with suspected chronic inflammatory conditions were excluded.

All the subjects underwent an US exam (EsaoteMyLab 70) of the clinically involved knee, performed by an expert sonographer that applied the new OMERACT criteria for the diagnosis of CPPD at the fibrocartilage (menisci) and hyaline cartilage of the affected knee (2).

Subsequently, a US-guided arthrocentesis was performed, and the synovial fluid was collected and analyzed by a compensated polarized light microscopy (AxioLab A.1 [Zeiss]) by an expert observer in order to assess the presence of CPP crystals. Both observers were blinded to clinical and to each other findings. The Chi-squared test was used to correlate the US and SFA findings.

Results: 49 patients ( 28 women) were enrolled in the study, with a mean age of $70,29$ yo (SD $\pm 10,93)$. 28 subjects were affected by CPPD at SFA and 26 patients 
were identified as affected by CPPD at US. In 4 patients, the SFA was positive and the US was negative, while in 2 patients the SFA was negative and the US positive. Using the chi-squared test, a very strong association was found between the exams, with a p-value $<0,0001$.

Conclusions: The new OMERACT US criteria for CPPD identification have already demonstrated to be reliable, considering the good to high kappa values yielded in previous multi-observer studies (2). This preliminary study, indicates that the new criteria seem to be also accurate for diagnostic purposes as they strongly correlate with the SFA for the presence of CPPD in knee joints. Further validation studies that will be able to assess the diagnostic accuracy of US are already in the research agenda of the OMERACT group "US in CPPD".

References:

[1] Filippou G, Adinolfi A, Cimmino MA, Scirè CA, Carta S, Lorenzini $S$, et al. Diagnostic accuracy of ultrasound, conventional radiography and synovial fluid analysis in the diagnosis of calcium pyrophosphate dihydrate crystal deposition disease. ClinExpRheumatol 2016.

[2] Filippou G, Scirè CA, Damjanov N et al. Definition and reliability assessment of elementary ultrasonographic findings in Calcium Pyrophosphate Deposition Disease. Results of an international multi-observer study by the OMERACT "US in CPPD" sub-task force. J Rheumatol, in press.

Disclosure of Interest: None declared

DOI: 10.1136/annrheumdis-2017-eular.5164

\section{FRI0638 COMPARISON OF ULTRASOUND AND MRI IN THE DIAGNOSIS OF PROXIMAL AND DISTAL BICEPS TENDON PATHOLOGY}

D. Huguet, C. Darrieutort-Laffite, B. Le Goff. Hopital Hotel Dieu Et Hme, Nantes Cedex 1, France

Background: Disorders of the long head of the biceps brachii tendon (LHBt) are commonly recognized as a source of shoulder pain. Ultrasound (US) is thought to be of limited value in the diagnosis of partial-thickness tear and non-tear abnormalities of the LHBt because of the difficulty to assess its intraarticular proximal portion. Brasseur recently described that placing the arm in extension/external rotation increased LHBt intra-articular portion visibility.

Objectives: The goal of this study was to determine if the systematic assessment of the intra-articular portion of the tendon, from the rotator interval to its glenoid insertion, with the arm placed in extension/external rotation could increase US sensitivity.

Methods: This was a cross-sectional study. All patients referred for the treatment of a rotator cuff disease (rupture, tendinopathy, calcific deposit) with an available MRI were included. US was performed blinded from the results of the clinical or MRI using a Sonosite Edge with a $6-13 \mathrm{MHz}$ probe. LHBt was studied at different level: in the bicipital groove, at the rotator interval, over the upper pole of the humerus head to its insertion on the superior glenoid tubercule. To increase the visibility of the proximal portion, we placed the arm in extension/external rotation as described by Brasseur [1]. Diagnosis of tendinopathy were tendon enlargement, hypoechogenicity and an increase in the interfibrillar distance. Subluxation or dislocation of the LHBt was defined as a partial or total loss of contact between the tendon and its groove. Tearing of tendon was defined as discontinuity or absence of tendon fibers. Fluid collection was defined as an anechoic ring around the tendon $>2 \mathrm{~mm}$. Abnormalities of the $\mathrm{LBHt}$ on MRI was retrieved from the report. $\mathrm{LBHt}$ abnormalities detected on arthroscopy were used as the gold standard.

Results: We included 129 patients, 57 female (44\%), and mean age 54 years (33-73). Seventy-five (58\%) had a rotator cuff tear and 54 (42\%) a tendinopathy. Arthroscopy found LHBt pathological changes in $39 \%$ of the case. The summary of the findings obtained with MRI, US (distal and proximal) and arthroscopy are summarised in table 1.

\begin{tabular}{lcccc} 
Table 1 & \multicolumn{1}{l}{} \\
\hline LHB & MRI & US distal & US proximal & Arthroscopy \\
\hline Normal & 99 & 90 & 111 & 78 \\
Subluxation & 15 & 6 & & 9 \\
Effusion & 12 & 30 & 6 & 6 \\
Tear & 3 & 3 & 12 & 2 \\
Flattened & & & & 30 \\
Thickening & & & & 1 \\
Nodular & & & & 3 \\
Inflammatory & & &
\end{tabular}

We calculated the sensitivity/specificity of MRI and US (at the proximal and distal level) in the detection of LHBt changes using arthroscopy as gold standard (Table 2).

Table 2

\begin{tabular}{lcccc}
\hline & Sensitivity & Specificity & PPV & PNV \\
\hline MRI & $52 \%$ & $96 \%$ & $90 \%$ & $73 \%$ \\
US proximal & $59 \%$ & $88 \%$ & $77 \%$ & $77 \%$ \\
US distal & $29 \%$ & $96 \%$ & $83 \%$ & $67 \%$ \\
\hline
\end{tabular}

Conclusions: US has a good specificity but a poor sensitivity in the detection of LHB tendon changes even when a systematic and carreful study of the proximal part of the tendon is undertaken. If detection of distal changes of the tendon in the inter-tubercular groove seems feasible with US, the involvement of the more proximal, intra-articular part of the tendon remains challenging. MRI sensitivity remains also poor. Overall, arthroscopy still remains the gold standard to detect LHB tendon intra-articular pathology.

References:

[1] Brasseur, The biceps tendons: from the top and from the bottom. Journal of ultrasound (2012) 15, 29-38.

Disclosure of Interest: None declared

DOI: 10.1136/annrheumdis-2017-eular.4468

\section{FRI0639 COULD BE THE ULTRASOUND STUDY OF PATIENTS WITH TROCHANTERIC PAIN USED TO CHOSE AN SPECIFIC THERAPEUTIC APPROACH?}

C.A. Guillen-Astete, M. Teran-Tinedo. Rheumatology Department, Ramon y Cajal University Hospital, Madrid, Spain

Background: Previous studies on tendinopathies of the hand (Quervain's tendinitis) and enthesis knee (anserine syndrome) have shown that some sort of ultrasound findings could predict the therapeutic response to specific treatments. Globally, acute inflammatory (power Doppler, synovial effusion) US findings predicts a better response to steroids injection than the chronic US findings (enthesophytosis, bone cortical irregularities).

Objectives: The aim of present study is to determine the probability of therapeutical success of different treatment approaches according to the presence of some specific US findings, in patients with trochanteric pain.

Methods: The image and clinical charts of patients who were assessed due to trochanteric pain between June 2015 and June 2016 in our clinic were reviewed. From those registries, dichotomic data about US findings were collected: Superficial bursitis, deep bursitis, cortical irregularities, enthesophytosis and power Doppler signal. The follow up data were collected from the electronic app or paper control form at day 6 to 9 from the original consultation. Successful treatment was interpreted as at least a reduction of $50 \%$ of the basal visual pain scale (rated from 1 to 10). Correlations with US findings were performed using single or composite variables.

Results: One hundred and twenty six registries of patients with trochanteric pain were included. From all of them, 119 belongs to female patients $(94.4 \%)$. The global US findings were as follows: 43 superficial bursitis, 39 deep bursitis, 47 cortical irregularities, 32 enthesophytosis and 6 power Doppler signal. Thirty seven steroids injection, 64 prescriptions of non steroideal antinflammatory drugs (NSAIDs) and 66 prescriptions of transdermal NSAIDs were performed.

Therapeutic success was achieved in $76.8 \%$ of patients who underwent an steroid injection in whom superficial or deep bursitis were identified in the US, and in $79.9 \%$ of patients who were treated with transdermal NSAIDs in whom enthesophytosis of cortical irregularities were identified.

By the other hand, patients treated with steroids injections in whom chronic US findings were achieved showed a failure treatment rate of $54.4 \%$. For those in whom any kind of bursitis were identified the failure treatment rate of transdermal NSAIDs was $65.5 \%$. Oral NSAIDs treatments were success in $35 \%$ of patients with any kind of bursitis and in $70 \%$ of patients with cortical irregularities or enthesophytosis.

Into the composite variables study we find that the treatment with steroid injection in patients with any kind of bursitis with independence of the presence of any chronic US findings had a Relative Risk (RR) of success of 2.66 compared to transdermal NSAID and a RR of 2.26 compared with oral NSAD treatment $(\mathrm{P}<0.001$ y $\mathrm{P}<0.01$, respectively). The only independent factor that demonstrated a $100 \%$ success treatment rate was the presence of power Doppler signal treated using steroid injection.

Conclusions: US findings are useful predictors of therapeutic response in patients with trochanteric pain. In general terms, we found that patients with US acute findings have an increased probability of treatment success when treated with steroid injection while those with US chronic findings have an increased probability of success when treated with NSAIDs (transdermal or systemic).

Disclosure of Interest: None declared

DOI: 10.1136/annrheumdis-2017-eular.6556

\section{FRI0640 STRUCTURAL DAMAGE PROGRESSION IN LUPUS ARTHRITIS: A PROSPECTIVE OBSERVATIONAL STUDY}

C. Tani ${ }^{1}$, A. Delle Sedie ${ }^{1}$, D. D'Aniello ${ }^{2}$, C. Stagnaro ${ }^{1}$, L. Carli $^{1,3}$, R. Vagelli ${ }^{1}$ F. Balestri ${ }^{1}$, S. Accogli ${ }^{4}$, D. Caramella ${ }^{4}$, M. Mosca ${ }^{1} \cdot{ }^{1}$ Rheumatology Unit, University of Pisa; ${ }^{2}$ Emergency Department, Azienda Ospedaliero Universitaria Pisana, Pisa: ${ }^{3}$ GenOMeC PhD, University of Siena, Siena: ${ }^{4}$ Diagnostic and Interventional Radiology, University of Pisa, Pisa, Italy

Background: Joint involvement is one of the main causes of chronic pain and disability in SLE patients (pts); despite arthritis in SLE is usually considered mild, joint erosions and deformities can be observed with significant impact on patient's quality of life. Imaging techniques are more sensitive than joint count in detecting synovitis and early joint damage.

Objectives: This study was aimed at evaluating the progression of joint damage in SLE and at evaluating predictive factors for damage accrual

Methods: Consecutive SLE pts with active hand-wrists synovitis (detected by joint 